Stefan Willitsch: Chemistry with controlled ions — Chap. 0 - 2016/5/17 - 10:55 — page 1

\title{
Chemistry with controlled ions
}

\author{
STEFAN WILLITSCH
}

Department of Chemistry, University of Basel, Klingelbergstrasse 80, 4056 Basel, Switzerland 
Stefan Willitsch: Chemistry with controlled ions — Chap. 0 - 2016/5/17 - 10:55 - page 2

2 
Stefan Willitsch: Chemistry with controlled ions — Chap. 0 - 2016/5/17 - 10:55 - page 3

\section{Contents}

\begin{tabular}{|c|c|}
\hline 1 & Chemistry with controlled ions 5 \\
\hline 1.1 & Introduction 5 \\
\hline 1.2 & Cooling and control of ions in the gas phase \\
\hline 1.2 .1 & \begin{tabular}{l|l} 
Ion trapping 6 \\
\end{tabular} \\
\hline 1.2 .2 & Cooling by collisions with neutrals \\
\hline 1.2 .3 & Laser and sympathetic cooling: Coulomb crystallization of ions \\
\hline$\overline{1.2 .4}$ & Selective preparation of quantum states 10 \\
\hline 1.2 .5 & $\begin{array}{ll}\text { Preparation of specific molecular conformations } & 12\end{array}$ \\
\hline 1.3 & Control of neutral molecules in the gas phase 13 \\
\hline 1.3 .1 & Cooling and control of the translational and internal molecular degrees of \\
\hline & freedom 13 \\
\hline 1.3 .2 & Conformer selection 15 \\
\hline 1.4 & Ion-molecule reaction dynamics: a brief outline \\
\hline 1.5 & $\begin{array}{ll}\text { Applications and examples } & 17 \\
\end{array}$ \\
\hline 1.5 .1 & Translationally cold ion-molecule collisions \\
\hline 1.5 .2 & Single-ion chemistry 21 \\
\hline 1.5 .3 & Energy- and state-controlled reactions 22 \\
\hline 1.5 .4 & Conformationally controlled ion-molecule reactions \\
\hline 1.6 & Conclusions and outlook 26 \\
\hline
\end{tabular}


Stefan Willitsch: Chemistry with controlled ions — Chap. 0 - 2016/5/17 - 10:55 — page 4

4 
Stefan Willitsch: Chemistry with controlled ions — Chap. 1 - 2016/5/17 - 10:55 — page 5

\section{Chemistry with controlled ions}

\section{1}

\section{Introduction}

Over the past years, the development of experimental methods for the manipulation of atoms and molecules in the gas phase has made impressive progress. The starting point for these developments was the advent of laser cooling of atoms in the 1970s and 1980s, which paved the way for the preparation of trapped ensembles of ultracold atoms and atomic ions [1]. These experimental breakthroughs initiated many exciting applications and new research directions in atomic physics, quantum science, and precision measurements [2, 3, 4].

Inspired by the successes with atoms, a variety of methods for the cooling of molecules and the preparation of cold molecular samples have been conceived since the mid-1990s. Techniques such as Stark deceleration [5], Zeeman deceleration [6] 7], Rydberg deceleration [6], photoassociation [8, 9], magnetic association [10, 11], velocity selection [12] and optical deceleration [13] enable the preparation of translationally cold molecules with kinetic energies corresponding to a few Kelvin down to hundreds of nanokelvin with exquisite quantum-state selectivity for some of these approaches. These developments have been reviewed extensively in the recent past [14, 15, 10, 16, 17, 18, 19, 20, 21, 22, 11, 23, 9, 24, 25, 5, 26, 27].

The ability to cool implies a large degree of control over the motion of molecules. Consequently, in recent years a new focus has developed to employ these techniques for the precise control of molecular motions with the objective to precisely control molecular collisions and chemical reactions [28, 29, 17, 30, 31, 32]. As opposed to coherent-control schemes which aim at shaping wavefunctions with short laser pulses [33], the approach discussed here concentrates on the precise manipulation of the translational motion, the position in space, the internal quantum state and energy as well as the geometric structure of the reaction partners using external electric, magnetic and optical fields. The ultimate objective of these efforts is to gain a comprehensive control over all dynamic properties of the collision partners, at the level of single molecules, to elucidate in detail chemical dynamics and reaction mechanisms as well as to control chemical processes. This approach can be viewed as taking to the extreme the control philosophy traditionally adopted in synthetic chemistry in 
Stefan Willitsch: Chemistry with controlled ions — Chap. 1 - 2016/5/17 - 10:55 — page 6

6

which reaction conditions are modified by changing the temperature, the pressure and the reactant concentrations and by using catalytic agents in order to influence the outcome of chemical reactions.

The present chapter focuses on the application of controlled-molecules techniques for precise studies of ion-molecule reactions in the gas phase. Section 1.2 and 1.3 gives an overview over the most important techniques presently used to control the translational motion, internal quantum states and structural properties of molecular ions and neutral molecules in the gas phase. Section 1.4 gives a brief outline of salient concepts of ion-molecule reaction dynamics which are relevant for the present discussion. In Section 1.5, some illustrative examples are presented in which coldand controlled-molecules techniques have been used to study the mechanisms and dynamics of ionic reactions.

\section{2}

\section{Cooling and control of ions in the gas phase}

\subsection{1}

\section{lon trapping}

Many state-of-the-art techniques for the control of ions rely on trapping. Among the instruments most widely used for the confinement of ions are linear radiofrequency (RF) traps in which an assembly of electrodes is arranged in a quadrupolar [34, 35, 36] or higher-order multipolar [37, 38] configuration. Charged particles are dynamically trapped in the center of these devices by the application of time-varying and static voltages to the electrodes.

The functionality of an RF ion trap is illustrated with the schematic of a typical linear quadrupole trap shown in Fig. 1.1 (a) [24]. The quadrupolar arrangement of the electrodes ensures the generation of a harmonic electrostatic potential in the center of the trap. The electrodes are separated into segments to allow the application of time-varying and static voltages. RF voltages $V_{\mathrm{RF}}=V_{0} \cos \left(\Omega_{\mathrm{RF}} t\right)$, with $V_{0}$ the $\mathrm{RF}$ amplitude and $\Omega_{\mathrm{RF}}$ the angular RF frequency, are applied to all segments of each electrode with a phase difference of $180^{\circ}$ between adjacent electrodes. The resulting time-varying electric potential ensures a dynamic trapping of the ions in the $(x, y)$ plane perpendicular to the longitudinal trap axis [34, 37]. The application of static voltages to the outermost segments of each electrode enables the confinement of the ions in the longitudinal trap direction $z$.

The classical motion of a single ion in a quadrupole RF trap follows a set of Mathieu equations [34, 39, 35]

$$
\frac{d^{2} u}{d t^{2}}+\frac{\Omega_{\mathrm{RF}}^{2}}{4}\left(a_{u}+2 q_{u} \cos \left(\Omega_{\mathrm{RF}} t\right)\right) u=0,
$$

where $u$ is any of the cartesian coordinates $x, y, z$. The Mathieu parameters $a_{u}$ and 
(a)

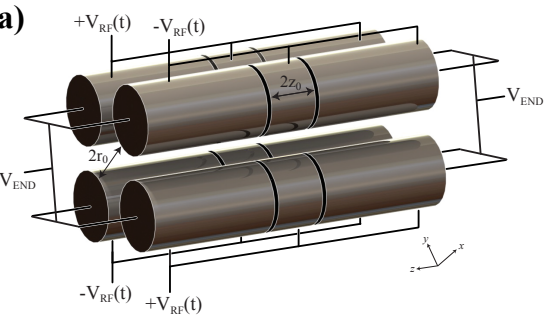

(c)

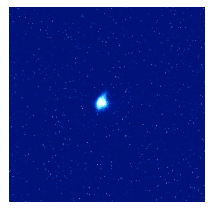

(b)

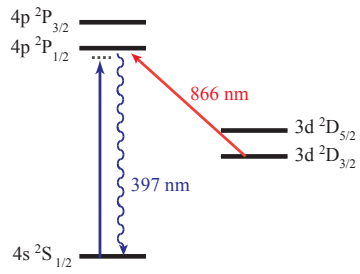

(d)

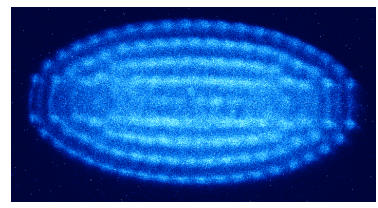

Figure 1.1 Linear radiofrequency (RF) ion traps and Coulomb crystals. (a) Schematic representation of a linear RF ion trap consisting of four cylindrical electrodes arranged in a quadrupolar configuration. The electrodes are sectioned into segments for the application of RF voltages $V_{\mathrm{RF}}$ and static voltages $V_{\text {end }}$ to allow the confinement of charged particles in the center of the trap. (b) Laser cooling scheme for $\mathrm{Ca}^{+}$. See text for details. (c) False-colour fluorescence images of Coulomb crystals of laser-cooled $\mathrm{Ca}^{+}$ions. Left: a single $\mathrm{Ca}^{+}$ion, right: a large spheroidal Coulomb crystal consisting of several hundred ions. (d) Bi-component Coulomb crystal consisting of laser-cooled $\mathrm{Ca}^{+}$ions and sympathetically cooled $\mathrm{N}_{2}^{+}$ions. The $\mathrm{N}_{2}^{+}$ions form a non-fluorescing string of ions in the center and are only indirectly visible as a dark core of the crystal. Figures (a) and (b) adapted from Ref. [24].

$q_{u}$ are defined by ${ }^{1)}$

$$
\begin{aligned}
& a_{x}=a_{y}=-\frac{1}{2} a_{z}=-\kappa \frac{4 q V_{\mathrm{END}}}{m \Omega_{\mathrm{RF}}^{2} z_{0}^{2}}, \\
& q_{x}=-q_{y}=\frac{4 q V_{0}}{m \Omega_{\mathrm{RF}}^{2} r_{0}^{2}}, \quad q_{z}=0 .
\end{aligned}
$$

In Eqs. 1.2,$m$ and $q$ stand for the mass and the charge of the ion, respectively, $2 z_{0}$ is the distance between the endcaps, $\kappa$ is a geometry parameter, and $2 r_{0}$ is the distance between two diagonally opposed quadrupole rods as shown in Fig. 1.1 (a). The specific combinations of $a_{u}, q_{u}$ for which a stable confinement of ions is feasible are usually represented in terms of stability diagrams [35, 34, 39].

The dynamic confinement using RF voltages imprints a fast oscillating motion on the ions termed "micromotion". The amplitude of the micromotion, and therefore the ion kinetic energy, scales with the magnitude of the RF potential at the position of the ion. In an adiabatic approximation [37, 34], the average kinetic energy stored in the micromotion appears as an effective (pseudo)potential $\Phi^{*}$ which governs the

1) See Ref. [24] for a discussion of different definitions of the Mathieu parameters. 
Stefan Willitsch: Chemistry with controlled ions — Chap. 1 - 2016/5/17 - 10:55 - page 8

$8 \mid$

slow thermal (secular) motions of the ions in the trap. For quadrupolar traps, the pseudopotential assumes a harmonic form:

$$
\Phi^{*}(x, y)=\frac{1}{2} m\left(\omega_{x}^{2} x^{2}+\omega_{y}^{2} y^{2}\right)
$$

where $\omega_{x}$ and $\omega_{y}$ are the secular frequencies for the ion's motion along $x$ and $y$, respectively. In a linear trap like the one shown in Fig. 1.1 (a), $\omega_{x}=\omega_{y}$ holds in the absence of static fields which break the symmetry. The RF potential (and therefore the micromotion) vanishes along the central axis of the trap for symmetry reasons. Higher-order multipole traps exhibit shallower potentials close to the trap center and therefore offer a reduced micromotion, however, at the expense of a tight confinement of the ions [37].

\section{2 .2}

\section{Cooling by collisions with neutrals}

"Traditional" methods for the cooling of ions rely on collisions with neutral gas particles which affect both the internal and external motional degrees of freedom of the ions. Cooling of the internal degrees of freedom manifests itself by a confinement of the populations to the lowest quantum states. Cooling of the translational motion of the ion results in a compression of the velocity distribution of the ion ensemble.

For decades, cooling in adiabatic gas expansions has been a method of choice for preparing internally cold molecules [40] and molecular ions [41, 42]. An adiabatic expansion of a gas from a high-pressure into a low-pressure zone results in the formation of a molecular beam and leads to the cooling of the internal degrees of freedom of the molecules through the conversion of internal energy to kinetic energy by collisions in the expansion region. Rotational temperatures down to about $1 \mathrm{~K}$ can be achieved using state-of-the-art pulsed gas nozzles [43] and sufficiently high pressure differentials between the gas reservoir and the expansion chamber maintained at high vacuum. The adiabatic expansion also results in translational cooling by a compression of the velocity distribution in the moving frame of the gas. Thus, the translational energy spread and therefore the temperature is small in the moving frame, but the molecules are still fast in the laboratory frame. While the translational temperatures in the moving frame are often comparable to the rotational ones, the vibrational temperatures of the molecules can be significantly higher owing to less efficient cooling of the vibrational motion by collisions in the expanding gas.

Another important technique for the cooling of ions relies on their immersion into a cryogenic buffer gas, e.g., helium [37, 44]. In this approach, the ions have to be confined to a well defined region of space using a trap. The translational and internal temperatures achievable with this method are limited by the temperature of the buffer gas which is typically on the order of $10 \mathrm{~K}$. 
Stefan Willitsch: Chemistry with controlled ions — Chap. 1 - 2016/5/17 - 10:55 — page 9

\subsection{3}

\section{Laser and sympathetic cooling: Coulomb crystallization of ions}

Recently, advanced methods such as laser and sympathetic cooling have been developed which enable to reach ion translational temperatures in the millikelvin regime, i.e., more than three orders of magnitudes lower than achievable with the collisional methods discussed above. These techniques have already been treated in detail elsewhere [1, 45, 46, 47, 24, 20] and shall briefly be summarized here.

Laser cooling of a particle is based on the removal of kinetic energy by the transfer of momentum imparted in the repeated scattering of photons from a laser beam [1, 45]. Since its introduction in the late 1970s [48, 49], it has become a workinghorse technique in atomic physics. It is applicable to systems with a sufficiently simple energy level structure which lends itself to the implementation of closed optical cycling transitions, i.e., so that the population is not lost during the repeated scattering of photons. As an example, a schematic level scheme illustrating the laser cooling of $\mathrm{Ca}^{+}$ions is shown in Fig. 1.1 (b). In this case, laser cooling is performed on the $4 s^{2} S_{1 / 2} \rightarrow 4 p^{2} P_{1 / 2}$ transition at $397 \mathrm{~nm}$. The laser frequency is slightly red-detuned from the resonance frequency of the transition to ensure that particles with a velocity component antiparallel to the laser beam preferentially absorb photons and are thus cooled (Doppler laser cooling). For neutral atoms, six laser beams in an optical molasses configuration, i.e., two beams counterpropagating on each coordinate axis, are necessary to achieve the cooling of all translational degrees of freedom. For large ensembles of ions in trap, cooling with a laser beam along a single direction is in principle sufficient because the Coulomb interaction between the particles couples all translational degrees of freedom.

For Doppler laser cooling, the limiting temperature $T_{D}$ can be achieved at an optimal detuning from resonance of $\Delta=-\gamma / 2$, where $\gamma$ is the natural linewidth of the cooling transition, and is given by [1, 45]

$$
T_{D}=\frac{\hbar \gamma}{2 k_{\mathrm{B}}} .
$$

For the laser cooling scheme of $\mathrm{Ca}^{+}$depicted in Fig. 1.1 (b), one obtains $T_{D}=$ $0.5 \mathrm{mK}$. From the excited state, spontaneous emission can occur back to the ground state or to the $3 d^{2} D_{3 / 2}$ level which cannot decay to the ground state by dipoleallowed optical transitions thus disrupting the laser-cooling cycle. Therefore, another laser beam at $866 \mathrm{~nm}$ is required to repump population back to the $4 p^{2} P_{1 / 2}$ state. Suitable systems for laser cooling include alkali atoms [45], alkaline earth ions [24], selected complex atoms such as erbium and dysprosium [50, 51] and a number of molecules exhibiting electronic transitions with near-diagonal Franck-Condon factors [52, 53].

Laser cooling of ions is typically performed in quadrupole ion traps which afford a tight trapping potential for charged particles (see Fig. 1.1) (a)). The tight confinement of the ions ensures their concentration in a small volume of space to facilitate overlap with the cooling laser beams and thus efficient cooling. The cooling of the ions under these conditions results in their spatial localization to form an ordered structure 
Stefan Willitsch: Chemistry with controlled ions — Chap. 1 - 2016/5/17 - 10:55 - page 10

referred to as a "Coulomb crystal" [46, 47, 24, 27]. Coulomb crystallization occurs when the ratio of the Coulomb repulsion energy between the ions and their thermal energy, the so-called plasma-coupling parameter, exceeds a value of about 150-170 [54, 55]. Typically, under tight trapping conditions in which the distances between the ions are on the order of ten microns, this criterion implies cooling of the ions down to tens of millikelvins which can readily be achieved with Doppler laser cooling.

Fig. 1.1 (c) shows false-color images of Coulomb crystals obtained by monitoring the fluorescence generated during laser cooling of $\mathrm{Ca}^{+}$ions. As can be seen from the images, individual ions can be observed, and therefore also addressed and manipulated, under these conditions. Consequently, the position of every single particle and by virtue of Eq. 1.3 its average micromotion energy is well defined 2$)$

As laser cooling is only applicable to a limited range of molecules [53], alternative methods resulting in the Coulomb crystallization of molecular ions need to be established. Over the past 15 years, sympathetic cooling has emerged as the method of choice for this purpose. In this approach, laser-cooled atomic ions are simultaneously trapped with molecular ions. The latter are cooled by collisions with the former, leading to the formation of bi-component Coulomb crystals which contain both species (see Fig. 1.1(d)). As this method relies on the transfer of momentum through collisions between charged species, it is extremely versatile and has been applied to molecular ions ranging in size from diatomics [57, 58] to small proteins [59]. To optimize the momentum transfer during collisions and thus cooling, the coolant and the cooled species should have a similar mass-to-charge-ratio [60]. In contrast to collisional cooling with neutral particles described in Sec. 1.2.3 sympathetic cooling with ions is mediated by collisions dominated by the Coulomb interaction and therefore does not appreciably affect the internal degrees of freedom of the molecules [61]. This problem can be solved by combining sympathetic cooling of the translational motion with cryogenic-buffer-gas cooling of the internal degrees of freedom, as has recently been shown in Ref. [62], or alternatively by using state-preparation techniques in combination with sympathetic cooling as detailed in the following section.

\section{2 .4}

\section{Selective preparation of quantum states}

Besides the control of the translational motion, preparation of the internal quantum state of molecular ions is a main prerequisite on the way to controlling chemical reactions. Since the 1980s, (multiphoton) photoionization has been a key method for preparing molecular ions in selected internal states [63]. Various variants of this approach have been implemented. Vibrationally state-selected ions have been produced by resonantly ionizing via specific vibrational states of a suitable Rydberg state of the neutral precursor molecule and capitalizing on diagonal Franck-Condon factors in the ionization step [64, 63, 65]. Rotational state selection has been achieved

2) Note, however, that in large crystals, thermal diffusion of the ions within the crystal structure can still be observed if the temperature is not sufficiently low [56. 
by pulsed-electric-field ionization of high Rydberg states of the neutral precursor molecule converging onto specific rotational states of the ion [66, 67]. Alternatively, ions can be produced in selected rotational states by photoionizing slightly above the lowest rotational ionization thresholds accessible from a well-defined intermediate state by photoionization propensity rules [68]. This approach has recently been applied to the state-selective preparation of $\mathrm{N}_{2}^{+}$ions in combination with sympathetic cooling of their translational motion to generate Coulomb-crystallized state-selected ions [58, 69]. The specific photoionization sequence used in these experiments is depicted in Fig. 1.2 (a).

An alternative approach for the internal-state preparation of molecular ions is "rotational laser cooling" [70, 72]. In this scheme, continuous-wave infrared excitation connecting specific rotational levels in the ground vibrational state (vibrational quantum number $v^{\prime \prime}=0$ ) and excited states $\left(v^{\prime}\right)$ of a molecule is implemented as illustrated with the example of $\mathrm{MgH}^{+}$in Fig. 1.2 (b). In this specific example, population is
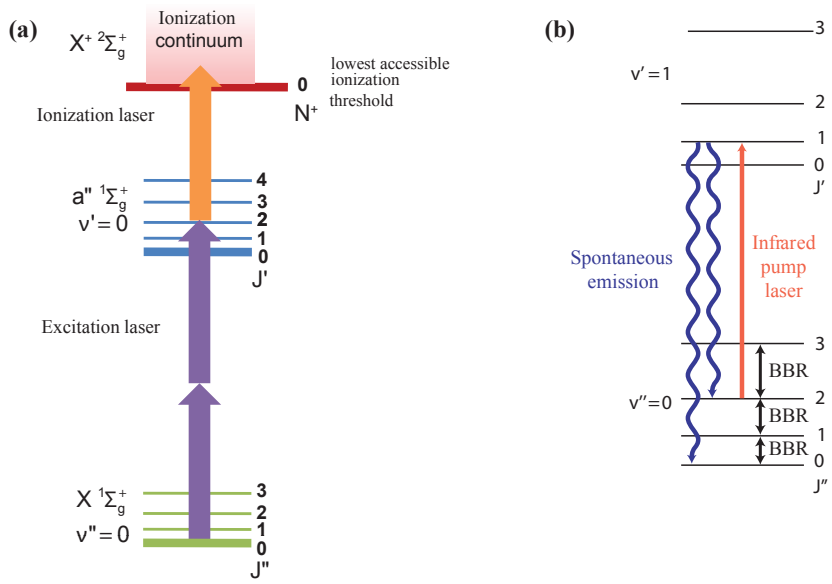

Figure 1.2 Preparation of molecular ions in selected rotational and vibrational states. (a) $\left[2+1^{\prime}\right]$ multiphoton threshold-photoionization scheme in $\mathrm{N}_{2}^{+}$from Ref. [58]. $J$ and $\mathrm{N}^{+}$ denote the rotational quantum numbers in the neutral and ionic states, respectively. An initial two-photon excitation step selects a specific rotational level (here with rotational quantum number $J^{\prime}=2$ ) in the excited $a^{\prime \prime}$ electronic state. Photoionization with an additional photon just above the lowest accessible rotational ionization threshold (corresponding to the cationic rotational ground state $N^{+}=0$ in this case) results in the generation of rotationally state-selected molecular ions. Adapted from Ref. [69]. (b) Rotational laser cooling in $\mathrm{MgH}^{+}$using an infrared laser irradiating the $v^{\prime \prime}=0, J^{\prime \prime}=2 \rightarrow v^{\prime}=1, J^{\prime}=1$ vibrational-rotational transition as implemented in Ref. [70]. The interplay between optical pumping on this transition (red arrow), fluorescence from the upper level back to rotational levels of the ground state (wavy arrows) and population redistribution between the rotational levels of the ground vibrational state by black-body radiation (BBR, black arrows) leads to an accumulation of the population in the absolute ground state $J=0$. See text for details. Figures adapted from Ref. [71]. 
pumped from the lower state (with rotational quantum number $J^{\prime \prime}=2$ ) to the upper state $v^{\prime}=1, J^{\prime}=1$. The excited state can fluoresce back to the $v^{\prime \prime}=0, J^{\prime \prime}=2$ state or the absolute rotational-vibrational ground state $v^{\prime \prime}=0, J^{\prime \prime}=0$ as dictated by electric-dipole selection rules. In addition, the rotational levels in $v "=0$ are coupled by the ambient black-body radiation (BBR) field which redistributes population between them. Thus, the $v^{\prime \prime}=0, J^{\prime \prime}=2$ level is constantly replenished with population from neighboring levels to be immediately pumped into the excited $v^{\prime}=1, J^{\prime}=1$ state from where it can fluoresce back into $v^{\prime \prime}=0, J^{\prime \prime}=0$ state. In the long run, this population-redistribution dynamics leads to an accumulation of population in the $v^{\prime \prime}=0, J^{\prime \prime}=0$ state.

The efficiency of this scheme can be improved by pumping on multiple rotationvibration transitions simultaneously. In experiments on $\mathrm{MgH}^{+}$with a single infrared pump laser, populations on the order of $40 \%$ could be achieved in the $J^{\prime \prime}=0$ state after a cooling time of about a minute. This represents a more than ten-fold increase of the population compared to the thermal value at room temperature [70]. In similar experiments in $\mathrm{HD}^{+}[72$ utilizing two infrared lasers pumping on different rotational-vibrational transitions, ground-state populations close to $80 \%$ could be achieved. Recently, broad-band laser-cooling techniques have been implemented [73] which utilize repeated femtosecond-laser pulses exciting a manifold of rotational levels simultaneously on a strong electronic transition. The excitation of the rotational ground state is excluded by spectral filtering of the laser pulses leading to an accumulation of population in the ground state through fluorescence from the excited electronic state. With this approach, it has been possible to reach ground state populations on the order of $95 \%$ after only $140 \mathrm{~ms}$ of cooling time in $\mathrm{AlH}^{+}$.

\section{2 .5}

\section{Preparation of specific molecular conformations}

The shape of a molecule is a key property influencing its chemical reactivity. Conformers, i.e., rotational isomers, are the dominant form of isomers of complex molecules. Hence, there is an need for techniques which enable the study of single molecular conformations. For the preparation of specific conformers of ions, several techniques have been developed in the past. Ion-mobility methods achieve the separation of different conformers, or families of conformers, from a mixture based on their geometrical cross sections for collisions while drifting through a buffer gas [74, 75, 76]. Alternatively, photoionization of specific conformers of neutral molecules has been successfully applied for the preparation of conformationally pure samples of ions. Here, conformer selection can either be achieved by single-photon excitation to high Rydberg states of a specific conformer followed by mass-analyzed threshold ionization (MATI) to produce the corresponding ion [77] or in resonant multi-photon ionization schemes in which a specific conformer is selected in the first excitation step to a suitable intermediate state [78]. 
Stefan Willitsch: Chemistry with controlled ions — Chap. 1 - 2016/5/17 - 10:55 - page 13

\section{3}

\section{Control of neutral molecules in the gas phase}

Controlling ion-molecule reactions necessitates control over both reaction partners, i.e., the ionic and neutral counterparts in a reaction. Similar to ions, cooling and translational control are in many cases two sides of the same coin also with neutrals. The last 15 years have seen impressive progress in the development of methods to control the translational motion of neutral molecules in the gas phase. These techniques have been reviewed extensively in the recent past, see, e.g., Refs. [17, 18, 20, $19,21,6,5,23,7,31,26,79$, and only a brief summary of the methods most relevant for the present purpose shall be given here.

\subsection{1}

\section{Cooling and control of the translational and internal molecular degrees of freedom}

A popular approach for producing translationally controlled molecules consists in the manipulation of the velocity of pulsed supersonic molecular beams using external electric, magnetic or optical fields. One of the most widely used methods is Stark deceleration which relies on the force $F_{S}$ generated by inhomogeneous electric fields

(a)

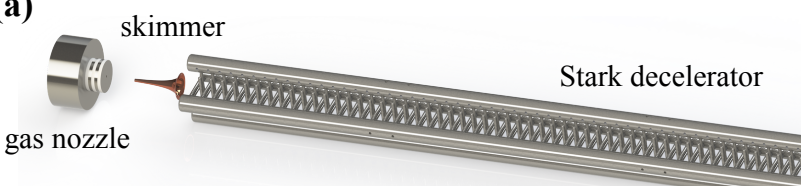

(b)

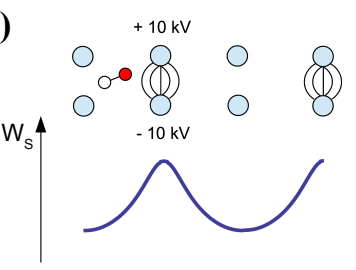

(c)

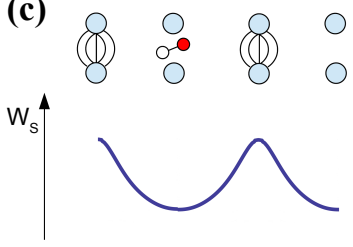

Figure 1.3 Principle of Stark deceleration. (a) Schematic representation of a Stark deceleration experiment consisting of a gas nozzle for producing a pulsed supersonic molecular beam which is coupled into an array of dipole electrodes. (b) Alternating pairs of electrodes are switched to high voltage creating a potential-energy $\left(W_{S}\right)$ barrier for molecules in low-field seeking Stark states. Thus, the molecules are slowed down when approaching the electrodes. (c) When the molecules approach the top of the barrier, the fields are switched off and applied to the next pair of electrodes. Repeated application of this scheme results in a gradual deceleration of the packet of molecules along the extent of the decelerator. 
Stefan Willitsch: Chemistry with controlled ions — Chap. 1 - 2016/5/17 - 10:55 — page 14

on polar molecules [80, 5, 32, 6, 19]:

$$
\vec{F}_{S}=-\vec{\nabla} W_{S}=\mu_{\mathrm{eff}} \vec{\nabla} E,
$$

where $W_{S}$ is the Stark energy of the molecule in a specific quantum state, $\mu_{\text {eff }}=$ $-\partial W_{S} / \partial E$ is the effective dipole moment of the molecule in this state and $E$ is the strength of the external electric field. $W_{S}$ is obtained by solving the relevant Stark problem of the system, see, e.g., Refs. [81, 82, 5]. Stark deceleration relies on molecules in low-field seeking Stark states, i.e., states which increase in energy with increasing electric field, so that a force is exerted which repels the molecules from regions of high field strength. In this scheme, a pulsed molecular beam of polar molecules such as $\mathrm{CO}, \mathrm{ND}_{3}$ or $\mathrm{OH}$ is injected into a linear array of dipole electrodes (Fig. 1.3 (a)). High voltages are applied when a packet of molecules approaches a given pair of electrodes. The Stark forces slightly decelerate the molecules while the have to surmount the potential-energy barrier posed by the electric field (Fig. 1.3 (b)). The fields are switched off before the molecules reach the top of the barrier to prevent their acceleration once they have passed the electrode stage and are applied to the next pair of electrodes to repeat the sequence (Fig. 1.3 (c)). Depending on the timing sequence of the application of the electric fields with respect to the position of the molecules within the electrode array, the molecules can be either decelerated or accelerated enabling the generation of velocity-controlled molecular beams [17]. This scheme can be used to bring the molecules to an effective stand still in the laboratory frame enabling their electric or magnetic trapping [83, 84].

Stark deceleration can only be employed to polar molecules showing a sufficiently strong Stark effect [85] which restricts the chemical diversity of the systems available to this method. The packets of molecules exiting a Stark decelerator possess a very high degree of state purity as the Stark force is very sensitive to the internal state of a molecule [5, 32]. Typically, the voltage switching sequence is optimized for the manipulation of a specific Stark state and molecules in other states are usually lost from the molecular beam while traversing the electrode array. Recently, the technique has been refined to utilize traveling electric traps generated in a linear array of ring electrodes which afford an improved control over the packet of decelerated molecules compared to conventional Stark deceleration [86].

A variant of this approach is the Stark deceleration of Rydberg atoms and molecules [87, 6, 79]. Because of the large dipole moments and concomitant large Stark forces achievable in Rydberg states, a single electrode stage is often sufficient to bring light molecules to an effective stand still. A chief advantage of this scheme is that it is applicable to polar and non-polar species alike, but requires the preparation of Rydberg states with sufficiently long lifetimes to cover at least the deceleration period (typically ms).

A related approach is Zeeman deceleration which utilizes the magnetic forces produced by inhomogeneous magnetic fields on paramagnetic atoms and molecules [88, 6, 7. In analogy to Stark deceleration, a pulsed molecular beam is coupled into a linear array of coils in which high currents are rapidly switched to create strong timevarying inhomogeneous magnetic fields used to decelerate (or accelerate) packets of molecules in low-field seeking Zeeman states. Like Stark deceleration, the scheme is 
highly state-specific and produces velocity-controlled molecular beams with a high state purity. Also, it has recently been extended to traveling magnetic traps which yield an improved control over the deceleration process [89, 90].

Another variation on the beam-deceleration theme is optical deceleration which relies on optical dipole forces produced by focussed laser beams [13, 91]. The potential energy $W_{O}$ of a molecule interacting with a far-off-resonant optical field is given by

$$
W_{O}(x, y, z, t)=-\frac{\alpha I(x, y, z, t)}{2 \epsilon_{0} c},
$$

where $\alpha$ is the effective polarizability of the molecule, $I(x, y, z, t)$ is the intensity of the optical field, $\epsilon_{0}$ is the vacuum permittivity and $c$ the speed of light. With laser radiation which is far red-detuned from all electronic transitions the particles are high-field seeking and drawn into the focus of the laser beam. Using the highly intense field of a focused nanosecond-pulse laser such as a Nd:YAG, it is possible to bring a packet of molecules delivered in a pulsed molecular beam to an effective stand still in a single laser shot. The scheme in principle applicable to virtually any polarizable species.

An alternative method to manipulate the velocity distribution of molecules is velocity filtering of an effusive molecular beam. In this approach, the slowest molecules from a thermal distribution are separated from the fast ones using the Stark forces generated in bent electric-multipole guides [12, 92, 93]. A schematic of a setup based on an electric-quadrupole guide is shown in Fig. 1.4 (a). The quadrupolar electric field inside the guide generates Stark forces on polar molecules which drive them towards the center when the molecules are in low-field seeking states. When the molecules approach the bend of the guide, only the slowest ones for which the Stark force exceeds the centrifugal force are retained whereas the faster molecules leave the bend and are lost from the guide.

This approach is capable of generating continuous beams of molecules with compressed velocity distributions corresponding to translational temperatures of a few Kelvin (Fig. 1.4 (b)). Like Stark deceleration, it is only applicable to polar species. Starting from a room-temperature source and a corresponding thermal distribution of rotational populations, typically a wide range of Stark states are filtered by the guide. As a consequence, the velocity-filtered beam of molecules is translationally cold, but rotationally warm [94, 92, 93]. This problem can be mitigated by feeding the guide with molecules from a cryogenic source which establishes a cooling of the internal degrees of freedom by collisions with a cryogenic buffer gas prior to velocity selection [95, 96].

\subsection{2}

\section{Conformer selection}

The selective study of individual conformers of complex neutral molecules in the gas phase is a challenging problem from an experimental perspective. In many cases, several conformers are populated at room temperature which may interconvert into 
Stefan Willitsch: Chemistry with controlled ions — Chap. 1 - 2016/5/17 - 10:55 - page 16

one another over low energy barriers. Recent years have seen impressive progress in the spectroscopic characterization of specific molecular conformations [97, 98, 99] and in the characterization of conformer-specific photodissociation dynamics $[77$. 78]. A common feature of these approaches is the adiabatic cooling of the molecules in a supersonic expansion in order to ensure the confinement of the populations to the energetically lowest conformers, thus reducing the conformational complexity, and to prevent their interconversion by thermal motion. Zwier and co-workers also achieved the "synthesis" and subsequent cooling of energetically excited molecular conformations by laser pumping infrared transitions promoting the interconversion between conformers in the high-pressure zone of a supersonic expansion [100, 101].

A method for the spatial separation of individual conformers in a molecular beam has recently been developed by Küpper, Stapelfeldt, Meijer and coworkers [102, 32]. The separation is based on differences in the mass-to-dipole moment ratios of different conformers. In this approach, a molecular beam containing a mixture of conformers is coupled into an electrostatic deflector consisting of two high-voltage electrodes creating a strong inhomogeneous electric field, see Fig. 1.8 (a) below for an illustration. Conformers with different dipole moments experience different Stark forces in the inhomogeneous field which deflect them perpendicularly to the beam axis. As a consequence, the conformers spatially separate and the molecular beam splits up into conformational components. The successful application of this scheme is subject to several prerequisites required to achieve a clean spatial separation of the conformers. First, the target molecule should not exhibit too many conformers which are populated in the molecular beam. Second, these conformers also need to exhibit sufficiently different ratios of their mass to their effective dipole moments to enable a clean separation. Thus far, this method has successfully been applied to medium-sized organic molecules like 3-aminophenol, 3-fluorophenol and indole-water clusters [32].

\section{4}

\section{Ion-molecule reaction dynamics: a brief outline}

Many ion-molecule reactions are effectively barrierless and are therefore governed by long-range intermolecular forces. In these cases, the reaction rates are determined by the efficiency of the capture of the reaction partners at long range. The longrange ion-molecule interaction potential $V(R)$, where $R$ is the separation of the center of masses of the two particles, is usually expressed in terms of a power series [103, 104, 105, 106, 107]. For ion-neutral interactions, this series can be formulated as

$$
V(R)=-\frac{q \mu_{D} \cos \beta}{R^{2}}-\frac{q Q\left(3 \cos ^{2} \gamma-1\right)}{2 R^{3}}-\frac{\alpha q^{2}}{2 R^{4}}+\ldots
$$

where $q$ is the charge of the ion, $\mu_{D}$ is the permanent dipole moment of the neutral molecule, $\beta$ is the angle between $\vec{\mu}_{D}$ and $\vec{R}, Q$ is the quadrupole moment of the neutral molecule, $\gamma$ is the angle between the axis of $Q$ and $\vec{R}$, and $\alpha$ is the isotropic 
polarizability of the neutral molecule. The terms in the order given in Eq. 1.7 correspond to the interaction of the charge of the ion with the permanent dipole moment, with the permanent quadrupole moment and with the induced dipole moment of the neutral molecule. The last term which scales with $R^{-4}$ is often called the Langevin interaction and is the leading term for an ion interacting with a structureless neutral particle, e.g., an atom in an $S$ state.

A long-range potential of the form of Eq. 1.7 is generally attractive and barrierless. The only barrier that then plays a role is the centrifugal barrier which arises from non-zero collisional angular momentum $L$. The centrifugal potential is given by $V_{\text {cent }}=L^{2} / 2 \mu R^{2}$, where $\mu$ is the reduced mass of the collision, and needs to be added to the interaction potential. Classically, the collisional angular momentum is given by $L=\mu v b$, where $v$ is the collision velocity and $b$ is the impact parameter. As ion neutral-interaction potentials are long range according to Eq. 1.7 most collisions occur at large impact parameters and therefore with large angular momentum.

The classical capture rate is given by all collisions which have enough kinetic energy to surmount the centrifugal barrier and lead to inward-spiraling trajectories which bring the particles close enough to react [108, 109]. For a charge-induced dipole interaction, this leads to the well-known Langevin rate constant $k_{\mathrm{L}}=2 \pi \sqrt{\alpha q^{2} / \mu}$ which is independent of the collision energy. More advanced capture models such as adiabatic capture theory [107, 110, 111] or the statistical adiabatic channel model [112, 105, 106] also take into account the effects of the quantum rotation of the neutral molecule while preserving a classical treatment of the translational motion. For very low collision energies at which individual collisional partial waves are resolved, the relative motion of the collision partners cannot be treated classically anymore and quantum capture treatments have been formulated (see, e.g., Refs. [113, 114, 115]).

\section{5}

\section{Applications and examples}

\subsection{1}

\section{Translationally cold ion-molecule collisions}

The combination of techniques for the control of the velocities of ions and neutrals discussed in Secs. 1.2 and 1.3, respectively, recently enabled the implementation of reaction experiments with controlled relative velocities of the reaction partners down to the "cold regime" defined as $E_{\text {coll }} / k_{\mathrm{B}}<1 \mathrm{~K}$. At low collision energies, the reaction dynamics often becomes very sensitive to subtleties of the underlying potential energy surface which opens up new possibilities to study reaction mechanisms and intermolecular interactions. The following examples may serve as an illustration.

In Ref. [47], the combination of an electrostatic quadrupole-guide velocity selector with an ion trap was introduced for studies of reactions between translationally cold neutrals and Coulomb-crystallized ions. A schematic of the setup is displayed in Fig. 1.4 (a). With this approach, collision energies corresponding to $\approx 5-100 \mathrm{~K}$ could be reached in ion-molecule collisions by tuning the velocity distribution of the 
Stefan Willitsch: Chemistry with controlled ions - Chap. 1 - 2016/5/17 - 10:55 - page 18

neutrals exiting the velocity filter and/or varying the size and shape of the Coulomb crystals in the trap [92].

First experiments focussed on reactions of laser-cooled $\mathrm{Ca}^{+}$ions with $\mathrm{CH}_{3} \mathrm{~F}$, $\mathrm{CH}_{2} \mathrm{~F}_{2}$ and $\mathrm{CH}_{3} \mathrm{Cl}$ [47, 116]. The rates of these reactions were determined from monitoring the decrease of the number $N$ of $\mathrm{Ca}^{+}$ions in the Coulomb crystals as

(a)

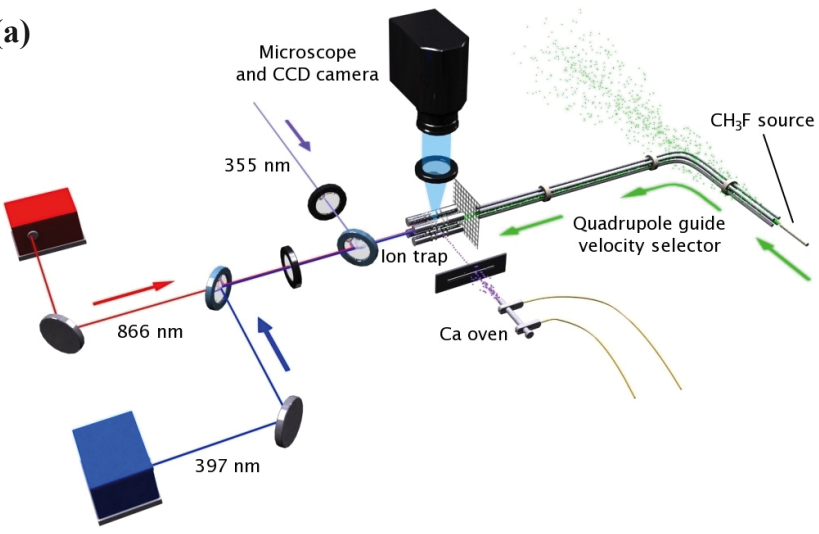

(b)

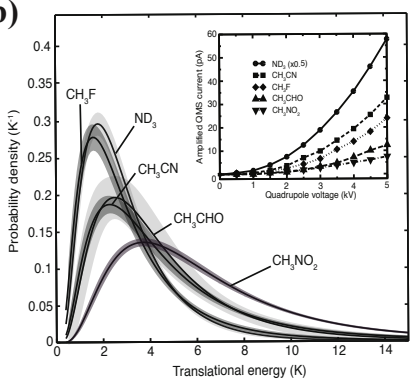

(c)

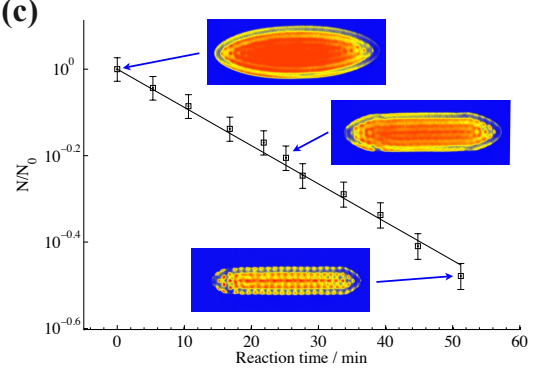

Figure 1.4 Reactions of cold ions with velocity-selected molecules. (a) Schematic of an experimental setup for studying translationally cold ion-molecule reactions. An electrostatic quadrupole-guide velocity selector produces a continuous beam of translationally cold polar molecules. The molecular beam enters a linear RF ion trap containing Coulomb crystals of cold ions. Laser beams for cooling and generation of $\mathrm{Ca}^{+}$ ions are labelled by their wavelengths $397 \mathrm{~nm}, 866 \mathrm{~nm}$ and $355 \mathrm{~nm}$. Adapted from Ref. [47]. (b) Translational energy distributions of various polar molecules which have been velocity-filtered by the electrostatic guide at a voltage of $V= \pm 4 \mathrm{kV}$ applied to the quadrupole electrodes. Inset: Averaged signal of polar molecules detected after the velocity filter at different quadrupole voltages. Adapted from Ref. [92]. (c) Rates of chemical reactions between cold $\mathrm{Ca}^{+}$ions and cold $\mathrm{CH}_{3} \mathrm{~F}$ molecules determined by monitoring the decrease of the volume of the Coulomb crystals, reflecting the decrease of the number $\mathrm{N}$ of $\mathrm{Ca}^{+}$ions, as a function of the reaction time. The bimolecular rate constant of the reaction is determined from a fit (solid line) of the experimental data to an integrated pseudo-first-order rate law, see text for details. Adapted from Ref. [16]. 
a function of the reaction time $t$ (Fig. 1.4(c)). Because of the excess of neutral molecules in comparison to the ions, the reactions show pseudo-first-order kinetics according to the integrated rate law $\ln N(t) / N(t=0)=-k n_{M} t$, where $k$ is the bimolecular rate constant and $n_{M}$ the number density of neutral molecules in the velocity-filtered beam. From a fit of the experimental data to the integrated rate law (solid line in Fig. 1.4 (c)), the rate constant $k$ can be determined if the neutral-molecule density $n_{M}$ is known.

Reactions of this type are initiated by an attack of the metal ion on a halogen atom on the neutral co-reactant leading to halogen abstraction from the molecule [117. The energy profile along the reaction coordinate is reminiscent of an $\mathrm{S}_{N} 2$ reaction [118] exhibiting a central energy barrier corresponding to the transition state of the halogen-abstraction step and minima in the entrance and exit channels of the reaction corresponding to ionic complexes [117]. Depending on the substitution pattern of the neutral reaction partner, the barrier maxima are located slightly above or below the energy of the reactants constituting dynamical bottlenecks which slow down the reaction on the ground-state potential-energy surface [116]. As during the experiment the $\mathrm{Ca}^{+}$ions are constantly laser cooled and therefore electronically excited, a fraction of the reactive collisions occurs with ions in the excited $(4 p){ }^{2} P_{1 / 2}$ or $(3 d)^{2} D_{3 / 2}$ states. In these cases, the reaction is initiated on an excited surface with rates close to the capture limit implying effectively barrierless processes. In cases such $\mathrm{Ca}^{+}+\mathrm{CH}_{2} \mathrm{~F}_{2}$ which exhibit high barriers on the lowest potential energy surface, the reaction rate observed in the experiment was completely dominated by the excited-state processes and that the effective reaction rate could be tuned by varying the populations in the excited states [116].

Even lower collision energies could be reached by immersing laser or sympathetically cooled ions into clouds of ultracold atoms [121, 122, 123, 124, 125, 126, 127. An example of such a "hybrid" trapping experiment is shown in Fig. 1.5(a) in which a linear RF ion trap has been combined with a magneto-optical trap (MOT) for ultracold neutral atoms, in this case ${ }^{87} \mathrm{Rb}[128,119,129]$. A MOT relies on a combination of six cooling laser beams in optical-molasses configuration and a quadrupolar magnetic field generated by two coils in anti-Helmholtz configuration to cool and confine atoms in its center. See, e.g., Refs. [130, 45, 1] for a detailed discussion of the working principle of a MOT. Apart from MOTs, [121, 122, 128, 126, 127, 131, 132], ion traps have also been combined with magnetic or optical dipole traps which allow the preparation of even colder samples of atoms down to temperatures of hundreds of $\mathrm{nK}[133,134,135]$.

As the neutral atoms are usually kept at temperatures $T<1 \mathrm{mK}$ while the ions are held at $T>1 \mathrm{mK}$ in the majority of these experiments, the collision energies are limited by the kinetic energies of the ions [136, 135]. The majority of the studies performed so far focused on studying sympathetic cooling of the ions by the atoms [133, 134, 131, 127, 137] and chemical processes between the cold ions and the atoms [128, 119, 138, 126, 139, 140, 141]. See Refs. [125, 124, 120] for reviews of recent studies.

An example demonstrating the potential of these type of cold-reaction studies for the elucidation of chemical dynamics can be found in Ref. [129] in which charge- 
Stefan Willitsch: Chemistry with controlled ions — Chap. 1 - 2016/5/17 - 10:55 - page 20

exchange reactions between translationally cold $\mathrm{N}_{2}^{+}$ions and and ultracold $\mathrm{Rb}$ atoms were studied in the hybrid trapping setup depicted in Fig. 1.5 (a). The $\mathrm{N}_{2}^{+}$ions were sympathetically cooled into the center of $\mathrm{Ca}^{+}$Coulomb crystal forming strings of ions located on the central symmetry axis of the trap, as shown in Fig. 1.1.(c), where the micromotion energy of the ions is minimized. In this way, collision energies as low as $E_{\text {coll }} / k_{\mathrm{B}} \approx 20 \mathrm{mK}$ could be reached. As the $\mathrm{Rb}$ atoms were constantly

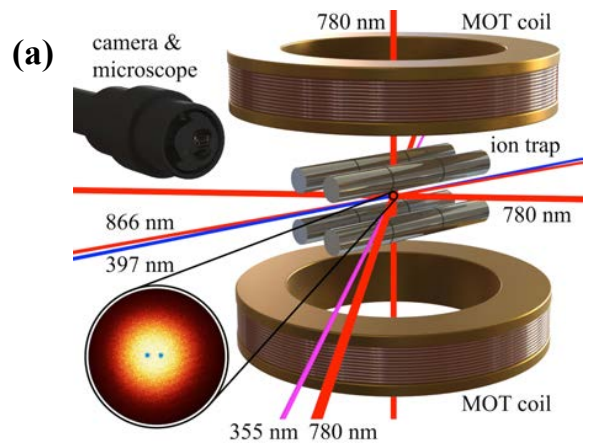

(b)

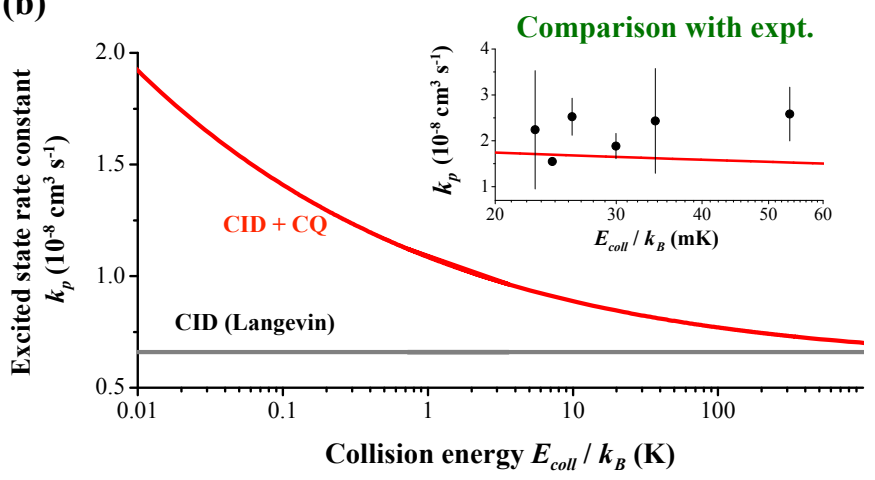

Figure 1.5 Cold ion-atom reactions. (a) Schematic of hybrid trapping experiment consisting of a linear RF trap for the laser and sympathetic cooling of ions integrated into a magneto-optical trap (MOT) for the laser cooling and trapping of neutral atoms, in the present case ${ }^{87} \mathrm{Rb}$. The MOT consists of two coils situated above and below the ion trap which are operated in an anti-Helmholtz configuration to create a quadrupolar magnetic field. In combination with six laser beams at $780 \mathrm{~nm}$ arranged in an optical-molasses configuration, the atoms are laser-cooled and confined to the center of the trap. The circular inset shows a false-color fluorescence image of two laser Cooled $\mathrm{Ca}^{+}$ions immersed into a cloud of trapped ultracold Rb atoms (yellow-red). Adapted from Ref. [119]. (b) Rate constant as a function of the collision energy for reactions of $\mathrm{N}_{2}^{+}$ions with $\mathrm{Rb}$ atoms in the excited $(5 p)^{2} P_{3 / 2}$ state predicted by classical capture theory taking into account the charge-induced dipole (CID, Langevin, black trace) and charge-induced-dipole plus charge-quadrupole (CQ) interactions (red trace). The inset shows a comparison of the predicted CID+CQ capture rate constants with experimental data. See text for details. Adapted from Ref. [120]. 
laser cooled during the experiment, collisions occurred with atoms in the ground $(5 s){ }^{2} S_{1 / 2}$ as well as the excited $(5 p)^{2} P_{3 / 2}$ state. It was found that the rate constant for collisions with $\mathrm{Rb}$ in the excited state exceeded the relevant Langevin rate by a factor $\approx 4$ in the collision energy interval between 20 and $60 \mathrm{mK}$ (Fig. 1.5 (b)). This finding could be rationalized in terms of a capture-limited process which is dominated by a long-range interaction between the charge of the ion and the permanent quadrupole moment of the $\mathrm{Rb}$ atom in its $(5 p)^{2} P_{3 / 2}$ state. In contrast to the Langevin rate constant which is independent of energy (Sec. 1.4), the rate constant arising from charge-quadrupole capture increases with decreasing energy so that it dominates the effective reaction rate in the cold regime (Fig. 1.5 (b)). At collision energies of a few hundred Kelvin, however, the effective rate constant is governed by the Langevin contribution and the effect of the charge-quadrupole interaction does not noticeably influence the reaction kinetics. These findings illustrate the value of controlled experiments at low collision energies for elucidating subtle details of intermolecular interactions which would otherwise be obscured in experiments at room temperature.

\section{5 .2}

\section{Single-ion chemistry}

One of the most intriguing aspects of the Coulomb-crystallization technique consists of the capability to perform experiments on single ions. In a chemical context, this offers the possibility to investigate chemical processes on the single-particle level, thus reaching an ultimate limit of sensitivity in chemical-reaction studies.

Pioneering experiments in this domain were performed by Drewsen and coworkers who devised methods for single-ion mass spectrometry [142], studying reactions on the single-ion level [143] and recycling the products of single-ion

(a)

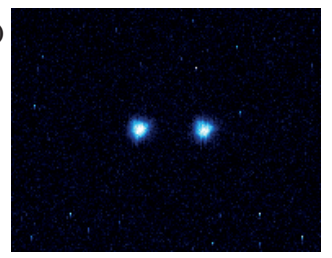

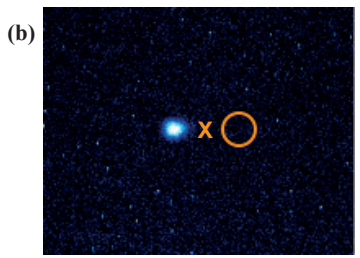

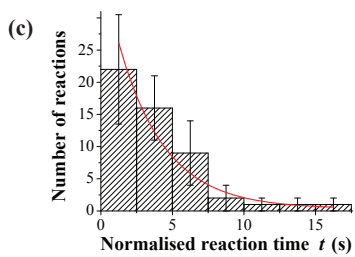

Figure 1.6 Single-ion reaction experiments. (a) A Coulomb crystal of two laser cooled $\mathrm{Ca}^{+}$ions prepared in the hybrid trapping experiment shown in Fig. 1.5 (a). (b) The same crystal after exposure to a cloud of ultracold $\mathrm{Rb}$ atoms. One of the $\mathrm{Ca}^{+}$ions has undergone a charge-transfer reaction and has been replaced by a non-fluorescing $\mathrm{Rb}^{+}$ ion (position indicated by the orange circle). The presence of the product ion manifests itself by the displacement of the remaining $\mathrm{Ca}^{+}$ion from the trap center (labeled with an $\mathrm{X}$ ) by the Coulomb repulsion between the ions. (c) Histogram of the time-to-reaction of $\mathrm{Ca}^{+}$ions with $\mathrm{Rb}$ obtained from 49 single-ion reaction experiments. The solid line shows a fit to a pseudo-first-order rate law. Adapted from Ref. [119]. 
Stefan Willitsch: Chemistry with controlled ions — Chap. 1 - 2016/5/17 - 10:55 — page 22

reactions in order to carry out repeated reactions with a single particle [144].

An example of a single-ion reaction study is shown in Fig. 1.6. In this experiment, which was performed in the hybrid-trap setup shown in Fig. 1.5(a), reactions of lasercooled $\mathrm{Ca}^{+}$ions with ultracold $\mathrm{Rb}$ atoms were studied. Fig. 1.6. (a) shows a Coulomb crystal of two laser cooled $\mathrm{Ca}^{+}$ions prepared before the reaction experiment. The $\mathrm{Ca}^{+}$ions are then immersed into a cloud of ultracold $\mathrm{Rb}$ atoms until one $\mathrm{Ca}^{+}$ion has reacted away. After reaction, the $\mathrm{Rb}$ cloud is removed and the product ion is sympathetically cooled by the remaining $\mathrm{Ca}^{+}$ion forming a two-ion bicomponent crystal (Fig. 1.6(b)). The product ion, the position of which is indicated by the orange circle in Fig. 1.6 (b), is not laser-cooled and therefore does not fluoresce, but its presence manifests itself by dislocating the remaining $\mathrm{Ca}^{+}$ion from the trap center (schematically indicated by an orange $X$ in Fig. 1.6(b)) via the mutual Coulomb repulsion. In the experiment shown, the product ion has been identified $\mathrm{as}^{\mathrm{Rb}}{ }^{+}$ using resonant-excitation mass spectrometry [142].

Rate constants for single-ion reactions can be determined by repeatedly measuring the time-to-reaction of single ions [119, 144]. A histogram of the number of ions $N$ reacting within a given time $t$ determined from 49 experiments on single $\mathrm{Ca}^{+}$is shown in Fig. 1.6 (c) [119]. The histogram was fitted to an exponential decay curve (red line) corresponding to a pseudo-first-order rate law (see above) from which the bimolecular rate constant $k$ was determined [119].

\subsection{3}

\section{Energy- and state-controlled reactions}

The ability to precisely control the translational and internal energies of reaction partners enables accurate studies of the energy balance of chemical processes. A proofof-principle experiment combining translational-energy- and quantum-state preparation of the reactants with state-resolved detection of the products was reported on the symmetric charge exchange reaction $\mathrm{N}_{2}^{+}+\mathrm{N}_{2} \rightarrow \mathrm{N}_{2}+\mathrm{N}_{2}^{+}$in Ref. [145]. In this work, $\mathrm{N}_{2}^{+}$ions were produced state-selectively by threshold photoionization of $\mathrm{N}_{2}$ from a molecular beam and sympathetically cooled into a Coulomb crystal as described in Sec. 1.2.4. The state-selected, translationally cold ions were then reacted with neutral $\mathrm{N}_{2}$ molecules from the same molecular beam (Fig. 1.7 (a)). Owing to the cooling in the adiabatic expansion, the neutral $\mathrm{N}_{2}$ molecules exhibited rotational temperatures $T_{\text {rot }}=10 \mathrm{~K}$ corresponding to $50 \%, 25 \%$ and $22 \%$ of the population in the $J=0,1$ and 2 rotational levels and a mean beam velocity of $787 \mathrm{~ms}^{-1}$. The $\mathrm{N}_{2}^{+}$ions were all produced in their rovibrational ground state forming a string of ions at the center of a $\mathrm{Ca}^{+}$Coulomb crystal where they exhibited an average kinetic energy $E_{\mathrm{kin}} / k_{\mathrm{B}}=10 \mathrm{mK}$. In this way, collisions between the ions and neutrals were investigated at a well-defined collision energy of $E_{\text {coll }}=0.045 \mathrm{eV}$ with reactions partners predominantly in their lowest rotational states. The rotational populations of the product $\mathrm{N}_{2}^{+}$ions were probed by a laser-induced charge transfer (LICT) technique [146]. Using another laser beam around $787 \mathrm{~nm}$, individual rotational-state populations were pumped to vibrationally excited states of the electronic ground state via excitation to the first excited electronic state of $\mathrm{N}_{2}^{+}$. From these vibrationally excited 
levels, the $\mathrm{N}_{2}^{+}$ions could undergo the vibrationally selective charge-transfer reaction $\mathrm{N}_{2}^{+}(v \geq 1)+\mathrm{Ar} \rightarrow \mathrm{N}_{2}+\mathrm{Ar}^{+}$which is energetically suppressed in the vibrational ground state $v=0$. The occurrence of LICT manifested itself in the disappearance of the $\mathrm{N}_{2}^{+}$ions from the center of the crystal.

In the experiment, the state populations of $\mathrm{N}_{2}^{+}$were monitored as a function of the exposure time to the molecular beam (Fig. 1.7(b)). It can be seen that the LICT efficiency (which is proportional to the population in the addressed rotational level)

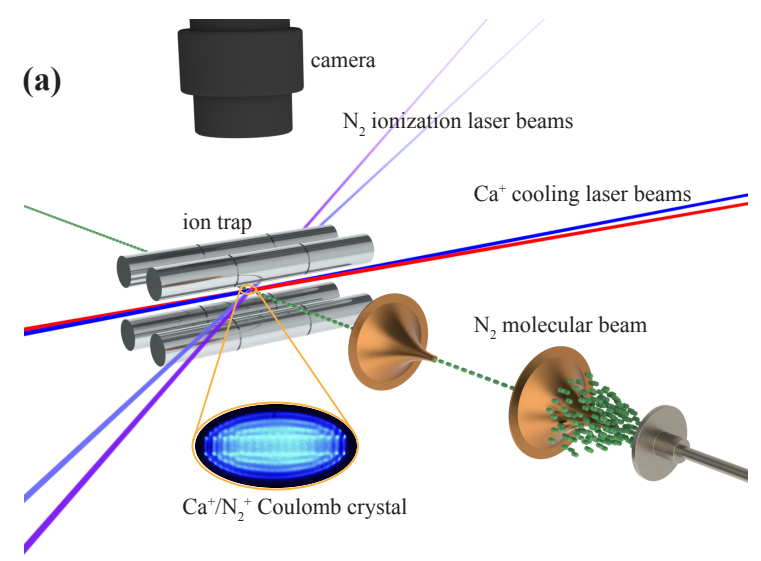

(b)

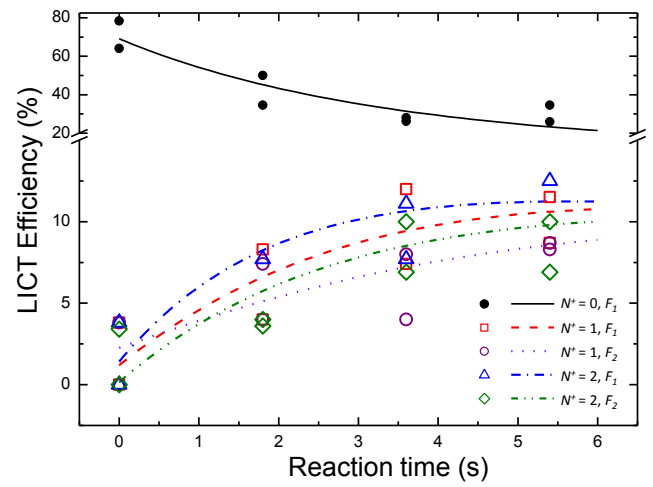

Figure 1.7 (a) Collision- and state-controlled ion-molecule reactions. (a) Schematic of the experimental setup. $\mathrm{N}_{2}^{+}$ions were prepared in their rotational-vibrational ground state using the threshold-photoionization scheme shown in Fig. 1.2 (a) and were sympathetically cooled into a Coulomb crystal of laser-cooled $\mathrm{Ca}^{+}$ions (inset). The state-selected $\mathrm{N}_{2}^{+}$ions were exposed to rotationally cold neutral $\mathrm{N}_{2}$ molecules introduced into the ion trap via a doubly skimmed supersonic molecular beam. (b) Dynamics of the populations in different spin-rotational states $N_{2}^{+}, F_{1,2}$ of sympathetically cooled $\mathrm{N}_{2}^{+}$ions as a function of the time of exposure to the molecular beam of neutral $\mathrm{N}_{2}$ molecules. The populations in the different states have been probed by laser-induced charge transfer (LICT) with Ar atoms. See text for details. Figures adapted from Ref. [145]. 
Stefan Willitsch: Chemistry with controlled ions — Chap. 1 - 2016/5/17 - 10:55 — page 24

decreases as a function of reaction time in the originally prepared rotational ground state $\mathrm{N}^{+}=0$, but increases in excited levels $N^{+} \geq 0$.

The $\mathrm{N}_{2}^{+}$ions in the Coulomb crystal are constantly exchanged by charge-transfer reactions with $\mathrm{N}_{2}$. The experimental results show that the charge transfer is not rotationally adiabatic and may produce ionic products in excited rotational states. With the help of reactive molecular dynamics simulations, this observation could be rationalized by the formation of a long-lived ion-molecule collision complex. Part of the initial collision energy is converted into rotational energy of the products after breakup of the complex. Thus, the increase in rotational energy of the products is a signature of translation-to-rotation energy transfer over the course of the reaction. The observation of these subtle energetic effects was only possible through the fine control over the collision and internal energies of the reaction partners in this experiment.

\section{5 .4}

\section{Conformationally controlled ion-molecule reactions}

In the past, studying conformational effects in chemical reaction dynamics has been notoriously difficult because of the challenges to isolate individual conformations in the gas phase, see Sec. 1.3.2 Besides matrix-isolation [148] and photodissociation [77. 78] studies, there are only few reports of conformationally-resolved bimolecular reactions in the gas phase, e.g., Ref. [149]. A new approach to unravel conformational effects in ion-molecule reactions has recently been presented by Chang et al. [147, 150]. It is based on the combination of an electrostatic conformer deflector for neutral molecules (Sec. 1.3.2 with a Coulomb-crystal experiment as depicted in Fig. 1.8 (a). Neutral conformers, as exemplified by the cis- and trans isomers of 3-aminophenol (AP) (Fig. 1.8(b)), are spatially separated in the inhomogeneous electric field of the deflector (a typical field distribution is shown in the inset in in Fig. 1.8 (a)) so that the molecular beam segregates into conformational components. Deflection profiles, i.e., density distributions for the two conformers at the point of the intersection of the molecular beam with the ion trap as a function of the extent of deflection, are shown in Fig. 1.8(b)). The relative intensities of the ion signals reflect the populations of the two conformers in the supersonic expansion. cis-AP has three times larger dipole moment than trans-AP $(2.33 \mathrm{D}$ and $0.77 \mathrm{D}$, respectively) so that the cis conformer is more strongly deflected than the trans conformer. At the particular deflector voltage $V=7.5 \mathrm{kV}$ of the experiment shown in Fig. 1.8(b)), the two conformers are not completely separated at the detection point, but regions in which either the trans or the cis conformers are dominant can be found at the smallest and largest deflection coordinates in Fig. 1.8 (b), respectively.

For measurements of conformationally dependent reaction rates, the molecular beam was directed at a Coulomb crystal of $\mathrm{Ca}^{+}$ions. Mass-spectrometric evidence [150] suggests that $\mathrm{Ca}^{+}$ions react with the aminophenol conformers predominantly in the $(4 p)^{2} P_{1 / 2}$ excited state by abstraction of one of the functional groups yielding $\mathrm{CaOH}^{+}$or $\mathrm{CaNH}_{2}^{+}$and the corresponding neutral radicals. The conformation 
Stefan Willitsch: Chemistry with controlled ions - Chap. 1 - 2016/5/17 - 10:55 - page 25

dependence of these processes is probed by tilting the molecular-beam apparatus so as to direct only a selected region of the deflected beam at the Coulomb crystal.
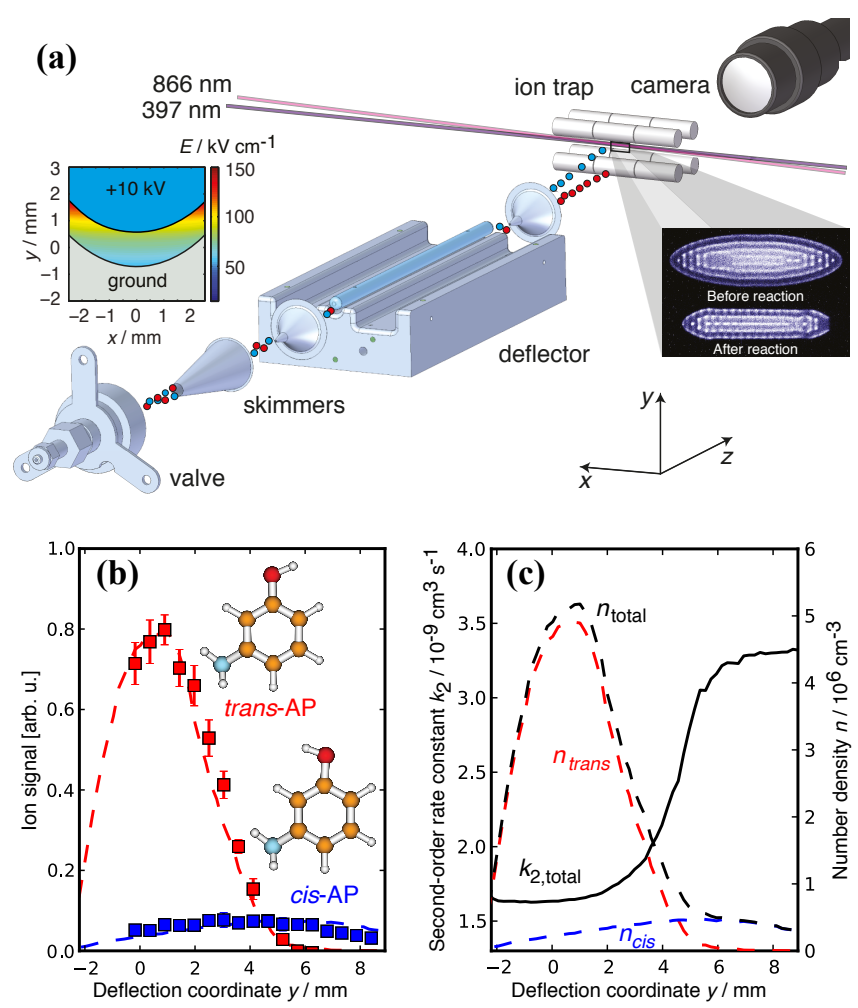

Figure 1.8 Conformationally controlled ion-molecule reactions. (a) Experimental setup consisting of an electrostatic deflector for conformers with different dipole moments entrained in a supersonic molecular beam. The inset on the left-hand side shows the electric field $E$ generated by the deflector electrodes. The spatially separated conformers (represented by the red and blue spheres) are directed at a tightly localized reaction target consisting of an ion Coulomb crystal in a linear RF trap. The inset on the right-hand side shows a Coulomb crystal of $\mathrm{Ca}^{+}$ions before and after reaction with conformers of 3-aminophenol. By tilting the molecular-beam assembly, reaction rates can be determined as a function of the coordinate of deflection for the individual conformers enabling to extract conformer-specific rate constants. (b) Density profiles of the deflected cis- and trans 3-aminophenol (AP) conformers as a function of the deflection coordinate at a deflector voltage of $V=7.5 \mathrm{kV}$. (c) Bimolecular rate constant $k_{2}$ for reactions of cis/trans-AP with laser-cooled $\mathrm{Ca}^{+}$ions as a function of the deflection coordinate. $n$ denotes the number density of the relevant conformers in the beam. The total rate constant is about a factor of 2 larger at deflection coordinates at which the cis-conformer prevails, indicating a higher reactivity for the cis-species. See text for details. All figures adapted from Ref. [147. 
Stefan Willitsch: Chemistry with controlled ions — Chap. 1 - 2016/5/17 - 10:55 - page 26

In this framework, the Coulomb crystal acts as tightly localized reaction target to provide a high spatial and therefore conformational resolution in reactions with the deflected conformers. By spatially scanning the deflected beam across the Coulomb crystal, the reaction rate constant as a function of the deflection coordinate and thus as a function of the conformational composition of the beam was measured (Fig. 1.8 (c)). One can see that the effective bimolecular reaction rate constant $k_{2, \text { total }}$ increases with deflection and is approximately twice as large at large deflection coordinates, where the cis conformer dominates, compared to small deflection coordinates, where the trans-conformer prevails.

The large absolute values of the rate constants $\left(k_{2}(\mathrm{cis})=3.2(13) \times 10^{-9} \mathrm{~cm}^{3} \mathrm{~s}^{-1}\right.$, $k_{2}($ trans $\left.)=1.5(6) \times 10^{-9} \mathrm{~cm}^{3} \mathrm{~s}^{-1}\right)$ suggests that the reaction kinetics is capture limited and governed by the properties of the long-range interaction between the reaction partners. An analysis within the framework of adiabatic capture theory [111] quantitatively confirmed the different values for the rate constants of the two conformers. It showed that the differences of the rate constants can be rationalized in terms of the different ion-dipole interactions exhibited by the two conformers: the cis conformer with the larger dipole has a stronger and therefore longer range interaction with an ion. This translates in to a larger capture impact parameter and therefore larger rate constant for this species.

\section{6}

\section{Conclusions and outlook}

Recent years have seen impressive progress in the control of ions in the gas phase. Molecular ions can be trapped and localized in space with precise control over their kinetic energies and rotational-vibrational quantum states. In combination with techniques for the control of neutral molecules, these methods have paved the way for studies of ion-molecule reactions unraveling exquisite details of their chemical dynamics.

The field is still in its infancy and promises a range of exciting developments for the future. A fascinating prospect is the study of the role of the hyperfine structure in chemical reactions. Recently, evidence has been found for the important role of the reactant hyperfine state in cold ion-neutral collisions [139]. For this purpose, it is highly desirable to develop hyperfine-state preparation techniques for molecular ions [151] as a prerequisite for studies of hyperfine effects in cold reactions. Another intriguing prospect is the application of state-control techniques developed in the realm of quantum logic to molecular ions [152, 153] which may ultimately also benefit chemical-reaction studies. So far, most experiments have focussed on small molecular systems, usually diatomics. The extension of the techniques discussed here to ever larger molecules and the study of polyatomic processes will be a prime objective for the coming years. In this context, improved capabilities for the analysis of the reaction products will be mandatory. For this purpose, several groups have recently developed ion traps coupled to time-of-flight mass spectrometers [154, 155]. Techniques for probing product state distributions, such as discussed in Sec. 1.5.3. 
Stefan Willitsch: Chemistry with controlled ions — Chap. 1 - 2016/5/17 - 10:55 — page 27

will also have to be refined and extended to a wider range of systems to gain a more comprehensive understanding of ion-molecule reactions dynamics.

\section{Acknowledgements}

This work is supported by the Swiss National Science Foundation, grant nrs. BSCGI0_157874 and 200021_156182 
Stefan Willitsch: Chemistry with controlled ions - Chap. 1 - 2016/5/17 - 10:55 - page 28

28 
Stefan Willitsch: Chemistry with controlled ions - Chap. 1 - 2016/5/17 - 10:55 - page 29

\section{References}

1 Metcalf, H.J. and van der Straten, P. (1999) Laser Cooling and Trapping, Springer, New York.

2 Cornell, E.A. and Wieman, C.E. (2002) Rev. Mod. Phys., 74, 875.

3 Wineland, D.J. (2013) Rev. Mod. Phys., 85, 1103.

4 Haroche, S. (2013) Rev. Mod. Phys., 85, 1083.

5 van de Meerakker, S.Y.T., Bethlem, H.L., Vanhaecke, N., and Meijer, G. (2012)

Chem. Rev., 112, 4828.

6 Hogan, S.D., Motsch, M., and Merkt, F. (2011) Phys. Chem. Chem. Phys., 13, 18705.

7 Narevicius, E. and Raizen, M.G. (2012) Chem. Rev., 112, 4879.

8 Jones, K.M., Tiesinga, E., Lett, P.D., and Julienne, P.S. (2006) Rev. Mod. Phys., 78, 483.

9 Ulmanis, J., Deiglmayr, J., Repp, M., Wester, R., and Weidemüller, M. (2012) Chem. Rev., 112, 4890.

10 Köhler, T., Gorál, K., and Julienne, P.S. (2006) Rev. Mod. Phys., 78, 1311.

11 Chin, C., Grimm, R., Julienne, P., and Tiesinga, E. (2010) Rev. Mod. Phys., 82, 1225.

12 Rangwala, S.A., Junglen, T., Rieger, T., Pinkse, P.W.H., and Rempe, G. (2003) Phys. Rev. A, 67, 043406.

13 Fulton, R., Bishop, A.I., and Barker, P.F. (2004) Phys. Rev. Lett., 93, 243004.

14 Bethlem, H.L. and Meijer, G. (2003) Int. Rev. Phys. Chem., 22, 73.

15 Hutson, J.M. and Soldán, P. (2006) Int. Rev. Phys. Chem., 25, 497.

16 Willitsch, S., Bell, M.T., Gingell, A.D., and Softley, T.P. (2008) Phys. Chem.
Chem. Phys., 10, 7200.

17 van de Meerakker, S.Y.T., Bethlem, H.L., and Meijer, G. (2008) Nat. Phys., 4, 595.

18 Krems, R.V., Stwalley, W.C., and Friedrich, B. (eds) (2009) Cold Molecules: Theory, Experiment, Applications, Boca Raton, CRC Press.

19 Schnell, M. and Meijer, G. (2009) Angew. Chem. Int. Ed., 48, 6010.

20 Bell, M.T. and Softley, T.P. (2009) Mol. Phys., 107, 99.

21 Carr, L.D., DeMille, D., Krems, R.V., and Ye, J. (2009) New J. Phys., 11, 055049.

22 Dulieu, O. and Gabbanini, C. (2009) Rep. Prog. Phys., 72, 086401.

23 Hutzler, N.R., Lu, H.I., and Doyle, J.M. (2012) Chem. Rev., 112, 4803.

24 Willitsch, S. (2012) Int. Rev. Phys. Chem., 31, 175.

25 Stuhl, B.K., Hummon, M.T., and Ye, J. (2014) Annu. Rev. Phys. Chem., 65, 501.

26 Jankunas, J. and Osterwalder, A. (2015) Annu. Rev. Phys. Chem., 66, 241.

27 Heazlewood, B. and Softley, T.P. (2015) Annu. Rev. Phys. Chem., 66, 475.

28 Krems, R.V. (2005) Int. Rev. Phys. Chem., 24, 99.

29 Krems, R.V. (2008) Phys. Chem. Chem. Phys., 10, 4079.

30 Quéméner, G. and Julienne, P.S. (2012) Chem. Rev., 112, 4949.

31 Lemeshko, M., Krems, R.V., Doyle, J.M., and Kais, S. (2013) Mol. Phys., 111, 1648

32 Chang, Y.P., Horke, D.A., Trippel, S., and Küpper, J. (2015) Int. Rev. Phys. Chem., 34, 557.

33 Shapiro, M. and Brumer, P. (2003) Principles of the Quantum Control of Molecular Processes, Wiley-Interscience, 
Stefan Willitsch: Chemistry with controlled ions — Chap. 1 - 2016/5/17 - 10:55 - page 30

Hoboken.

34 Major, F.G., Gheorghe, V.N., and Werth, G. (2005) Charged Particle Traps, Springer, Berlin and Heidelberg.

35 March, R.E. and Todd, J.F. (2005) Quadrupole Ion Trap Mass Spectrometry, John Wiley \& Sons, Hoboken, 2nd edn..

36 Leibfried, D., Blatt, R., Monroe, C., and Wineland, D. (2003) Rev. Mod. Phys., 75, 281.

37 Gerlich, D. (1992) Adv. Chem. Phys., 82, 1.

38 Wester, R. (2009) J. Phys. B, 42, 154001.

39 Drewsen, M. and Brøner, A. (2000) Phys. Rev. A, 62, 045401.

40 Scoles, G. (ed.) (1988) Atomic and Molecular Beam Methods, Oxford University Press, Oxford.

41 Smith, M.A. (1998) Int. Rev. Phys. Chem., 17, 35.

42 Rowe, B.R., Canosa, A., and Le Page, V. (1995) Int. J. Mass Spectrom. Ion Proc., 149/150, 573.

43 Even, U. (2015) Eur. Phys. J. Tech. Instr., $2,17$.

44 Gerlich, D. (1995) Ion-neutral collisions in a 22-pole trap at very-low energies. Physica Scripta, T59, 256.

45 Foot, C.F. (2005) Atomic Physics, Oxford University Press, Oxford.

46 Drewsen, M., Jensen, I., Lindballe, J., Nissen, N., Martinussen, R., Mortensen, A., Staanum, P., and Voigt, D. (2003) Int. J. Mass Spectrom., 229, 83.

47 Willitsch, S., Bell, M.T., Gingell, A.D., Procter, S.R., and Softley, T.P. (2008) Phys. Rev. Lett., 100, 043203.

48 Wineland, D.J., Drullinger, R.E., and Walls, F.L. (1978) Phys. Rev. Lett., 40, 1639.

49 Neuhauser, W., Hohenstatt, M., Toschek, P., and Dehmelt, H. (1978) Phys. Rev. Lett., 41, 233.

50 McClelland, J.J. and Hanssen, J.L. (2006) Phys. Rev. Lett., 96, 143005.

51 Lu, M., Youn, S.H., and Lev, B.L. (2010) Phys. Rev. Lett., 104, 063001.

52 Shuman, E.S., Barry, J.F., and DeMille, D. (2010) Nature, 467, 820 .

53 Isaev, T.A. and Berger, R. (2016) Phys. Rev. Lett., 116, 063006.

54 Pollock, E.L. and Hansen, J.P. (1973) Phys. Rev. A, 8, 3110.
55 Farouki, R.T. and Hamaguchi, S. (1993) Phys. Rev. E, 47, 4330.

56 Zhang, C.B., Offenberg, D., Roth, B., Wilson, M.A., and Schiller, S. (2007) Phys. Rev. A, 76, 012719.

57 Mølhave, K. and Drewsen, M. (2000) Phys. Rev. A, 62, 011401.

58 Tong, X., Winney, A.H., and Willitsch, S (2010) Phys. Rev. Lett., 105, 143001.

59 Offenberg, D., Zhang, C.B., Ch. Wellers, Roth, B., and Schiller, S. (2008) Phys. Rev. A, 78, 061401.

60 Baba, T. and Waki, I. (2002) Appl. Phys. $B, \mathbf{7 4}, 375$.

61 Bertelsen, A., Jorgensen, S., and Drewsen, M. (2006) J. Phys. B: At. Mol. Opt. Phys., 39, L83.

62 Hansen, A.K., Versolato, O.O., Klosowski, L., Kristensen, S.B., Gingell, A., Schwarz, M., Windberger, A., Ullrich, J., Lopez-Urrutia, J.R.C., and Drewsen, M. (2014) Nature, 508, 76-79.

63 Anderson, S.L. (1992) Adv. Chem. Phys., 82, 177.

64 Morrison, R.J.S., Conaway, W.E., Ebata, T., and Zare, R.N. (1986) J. Chem. Phys., 84, 5527.

65 Sage, A.D., Oliver, T.A.A., Dixon, R.N., and Ashfold, M.N.R. (2010) Mol. Phys., 108, 945.

66 Mackenzie, S.R. and Softley, T.P. (1994) J. Chem. Phys., 101, 10609.

67 Green, R.J., Qian, J., Kim, H.T., and Anderson, S.L. (2000) J. Chem. Phys., 113, 3002.

68 Willitsch, S. and Merkt, F. (2005) Int. J. Mass Spectrom., 245, 14.

69 Tong, X., Wild, D., and Willitsch, S. (2011) Phys. Rev. A, 83, 023415.

70 Staanum, P.F., Højbjerre, K., Skyt, P.S., Hansen, A.K., and Drewsen, M. (2010) Nat. Phys., 6, 271.

71 Willitsch, S. (2010) Nat. Phys., 6, 240.

72 Schneider, T., Roth, B., Duncker, H., Ernsting, I., and Schiller, S. (2010) Nat. Phys., 6, 275.

73 Lien, C.Y., Seck, C.M., Lin, Y.W., Nguyen, J.H.V., Tabor, D.A., and Odom, B.C. (2014) Nat. Commun., 5, 4783.

74 Shvartsburg, A.A. (2008) Differential Ion Mobility Spectrometry, CRC Press, Boca Raton.

75 Bohrer, B.C., Merenbloom, S.I., 
L.Koeniger, S., E.Hilderbrand, A., and Clemmer, D.E. (2008) Annu. Rev. Anal. Chem., 1, 293.

76 Papadopoulos, G., Svendsen, A., Boyarkin, O.V., and Rizzo, T.G. (2011) Faraday Discuss., 150, 243.

77 Park, S.T., Kim, S.Y., and Kim, M.S. (2002) Nature, 415, 306.

78 Kim, M.H., Shen, L., Tao, H., Martinez, T.J., and Suits, A.G. (2007) Science, 315, 1561.

79 Hogan, S.D. (2016) Eur. Phys. J. Tech. Instr., 3, 2.

80 Bethlem, H.L., Berden, G., and Meijer, G. (1999) Phys. Rev. Lett., 83, 1558.

81 Kroto, H.W. (1975) Molecular Rotation Spectra, John Wiley \& Sons, London.

82 Brown, J.M. and Carrington, A. (2003) Rotational Spectroscopy of Diatomic Molecules, Cambridge University Press.

83 van de Meerakker, S.Y.T., Vanhaecke, N., van der Loo, M.P.J., Groenenboom, G.C., and Meijer, G. (2005) Phys. Rev. Lett., 95 , 013003.

84 Sawyer, B.C., Lev, B.L., Hudson, E.R., Stuhl, B.K., Lara, M., Bohn, J.L., and Ye, J. (2007) Phys. Rev. Lett., 98, 253002.

85 Bethlem, H.L., Crompvoets, F.M.H., Jongma, R.T., van de Meerakker, S.Y.T., and Meijer, G. (2002) Phys. Rev. A, 65, 053416 .

86 Osterwalder, A., Meek, S.A., Hammer, G., Haak, H., and Meijer, G. (2010) Phys. Rev. A, 81, 051401.

87 Vliegen, E., Wörner, H.J., Softley, T.P., and Merkt, F. (2004) Phys. Rev. Lett., 92, 033005.

88 Vanhaecke, N., Meier, U., Andrist, M., Meier, B.H., and Merkt, F. (2007) Phys. Rev. A, 75, 031402.

89 Trimeche, A., Bera, M.N., Cromiéres, J.P., Robert, J., and Vanhaecke, N. (2011) Eur. Phys. J. D, 65, 263.

90 Akerman, N., Karpov, M., David, L., Lavert-Ofir, E., Narevicius, J., and Narevicius, E. (2015) New J. Phys, 17, 065015.

91 Fulton, R., Bishop, A.I., Shneider, M.N., and Barker, P.F. (2006) J. Phys. B: At. Mol. Opt. Phys., p. S1097.

92 Bell, M.T., Gingell, A.D., Oldham, J., Softley, T.P., and Willitsch, S. (2009) Faraday Discuss., 142, 73.
93 Bertsche, B. and Osterwalder, A. (2010) Phys. Rev. A, 82, 033418.

94 Motsch, M., Schenk, M., van Buuren, L.D., Zeppenfeld, M., Pinkse, P.W.H., and Rempe, G. (2007) Phys. Rev. A, 76, 061402.

95 van Buren, L.D., Sommer, C., Motsch, M., Pohle, S., Schenk, M., Bayerl, J., Pinkse, P.W.H., and Rempe, G. (2009) Phys. Rev. Lett., 102, 033001.

96 Twyman, K.S., Bell, M.T., Heazlewood, B.R., and Softley, T.P. (2014) J. Chem. Phys., 141, 024308.

97 Simons, J.P., Jockusch, R.A., Carcabal, P., Hünig, I., Kroemer, R.T., Macleod, N.A., and Snoek, L.C. (2005) Int. Rev. Phys. Chem., 24, 489.

98 de Vries, M.S. and Hobza, P. (2007) Annu. Rev. Phys. Chem., 58, 585.

99 Rizzo, T.R., Stearns, J.A., and Boyarkin, O.V. (2009) Int. Rev. Phys. Chem., 28, 481.

100 Dian, B.C., Longarte, A., and Zwier, T.S. (2002) Science, 296, 2369.

101 Dian, B.C., Clarkson, J.R., and Zwier, T.S. (2004) Science, 303, 1169.

102 Filsinger, F., Küpper, J., Meijer, G., Hansen, J.L., Maurer, J., Nielsen, J.H., Holmegaard, L., and Stapelfeldt, H. (2009) Angew. Chem. Int. Ed., 48, 6900.

103 Hirschfelder, J.O. and Meath, W.J. (1967) Adv. Chem. Phys., 12, 3.

104 Stone, A. (2013) The Theory of Intermolecular Forces, Oxford University Press, Oxford, 2nd edn.

105 Maergoiz, A.I., Nikitin, E.E., Troe, J., and Ushakov, V.G. (1996) J. Chem. Phys., 105, 6263

106 Maergoiz, A.I., Nikitin, E.E., Troe, J., and Ushakov, V.G. (1996) J. Chem. Phys., 105, 6270 .

107 Clary, D.C. (1985) Mol. Phys., 54, 605.

108 Gioumousis, G. and Stevenson, D.P. (1958) J. Chem. Phys., 29, 294.

109 Levine, R.D. (2005) Molecular Reaction Dynamics, Cambridge University Press, Cambridge.

110 Clary, D.C. (1995) Chem. Phys. Lett., 232, 267.

111 Stoecklin, T., Clary, D.C., and Palma, A. (1992) J. Chem. Soc. Faraday Trans., 88, 901.

112 Troe, J. (1987) J. Chem. Phys., 87, 2773. 
Stefan Willitsch: Chemistry with controlled ions — Chap. 1 - 2016/5/17 - 10:55 - page 32

113 Dashevskaya, E.I., Maergoiz, A.I., Troe, J., Litvin, I., and Nikitin, E.E. (2003) J. Chem. Phys., 118, 7313.

114 Gao, B. (2010) Phys. Rev. Lett., 104, 213201.

115 Gao, B. (2011) Phys. Rev. A, 83, 062712.

116 Gingell, A.D., Bell, M.T., Oldham, J.M., Softley, T.P., and Harvey, J.M. (2010) J. Chem. Phys., 133, 194302.

117 Harvey, J.N., Schröder, D., Koch, W., Danovich, D., Shaik, S., and Schwarz, H. (1997) Chem. Phys. Lett., 273, 164.

118 Mikosch, J., Trippel, S., Eichhorn, C., Otto, R., Lourderaj, U., Zhang, J.X., Hase, W.L., Weidemüller, M., and Wester, R. (2008) Science, 319, 183.

119 Hall, F.H.J., Eberle, P., Hegi, G., Raoult, M., Aymar, M., Dulieu, O., and Willitsch, S. (2013) Mol. Phys., 111, 2020.

120 Willitsch, S. (2015) Proc. Int. Sch. Phys. Enrico Fermi, 189, 255.

121 Smith, W.W., Makarov, O.P., and Lin, J. (2005) J. Mod. Opt., 52, 2253.

122 Grier, A.T., Cetina, M., Oručević, F., and Vuletić, V. (2009) Phys. Rev. Lett., 102, 223201.

123 Willitsch, S. (2014) arXiv: 1401.1699v1 [physics.atom-ph].

124 Sias, C. and Köhl, M. (2014) Quantum Gas Experiments, World Scientific Publishing, Singapore, p. 267.

125 Härter, A. and Hecker Denschlag, J. (2014) Contemp. Phys., 55, 33.

126 Rellergert, W.G., Sullivan, S.T., Kotochigova, S., Petrov, A., Chen, K., Schowalter, S.J., and Hudson, E.R. (2011) Phys. Rev. Lett., 107, 243201.

127 Ravi, K., Lee, S., Sharma, A., Werth, G., and Rangwala, S.A. (2012) Nat. Commun., 3, 1126.

128 Hall, F.H.J., Aymar, M., Bouloufa-Maafa, N., Dulieu, O., and Willitsch, S. (2011) Phys. Rev. Lett., 107, 243202.

129 Hall, F.H.J. and Willitsch, S. (2012) Phys. Rev. Lett., 109, 233202.

130 Raab, E.L., Prentiss, M., Cable, A., Chu, S., and Pritchard, D.E. (1987) Phys. Rev. Lett., 59, 2631.

131 Sivarajah, I., Goodman, D.S., Wells, J.E., Narducci, F.A., , and Smith, W.W. (2012) Phys. Rev. A, 86, 063419.

132 Haze, S., Hata, S., Fujinaga, M., and Mukaiyama, T. (2013) Phys. Rev. A, 87,
052715.

133 Zipkes, C., Palzer, S., Sias, C., and Köhl, M. (2010) Nature, 464, 388

134 Schmid, S., Härter, A., and Hecker Denschlag, J. (2010) Phys. Rev. Lett., 105, 133202.

135 Meir, Z., Sikorsky, T., Ben-shlomi, R., Akerman, N., Dallal, Y., , and Ozeri, R. (2016) arXiv, p. 1603.01810.

136 Cetina, M., Grier, A.T., and Vuletić, V. (2012) Phys. Rev. Lett., 109, 253201.

137 Rellergert, W.G., Sullivan, S.T., Schowalter, S.J., Kotochigova, S., Chen, K., and Hudson, E.R. (2013) Nature, 495, 490.

138 Hall, F.H.J., Aymar, M., Raoult, M., Dulieu, O., and Willitsch, S. (2013) Mol. Phys., 111, 1683.

139 Ratschbacher, L., Zipkes, C., Sias, C., and Köhl, M. (2012) Nat. Phys., 8, 649.

140 Härter, A., Krükow, A., Dei, M., Drews, B., Tiemann, E., and Hecker Denschlag, J. (2013) Nat. Phys., 9, 512.

141 Haze, S., Saito, R., Fujinaga, M., and Mukaiyama, T. (2015) Phys. Rev. A, 91 , 032709.

142 Drewsen, M., Mortensen, A., Martinussen, R., Staanum, P., and Sorensen, J.L. (2004) Phys. Rev. Lett., 93, 243201.

143 Staanum, P.F., Højbjerre, K., Wester, R., and Drewsen, M. (2008) Phys. Rev. Lett., 100, 243003.

144 Hansen, A.K., Sørensen, M.A., Staanum, P.F., and Drewsen, M. (2012) Angew. Chem. Int. Ed., 51, 7960.

145 Tong, X., Nagy, T., Yosa Reyes, J., Germann, M., Meuwly, M., and Willitsch, S. (2012) Chem. Phys. Lett., 547, 1.

146 Schlemmer, S., Kuhn, T., Lescop, E., and Gerlich, D. (1999) Int. J. Mass Spectrom., 185/186/187, 589 .

147 Chang, Y.P., Dlugolecki, K., Küpper, J., Rösch, D., Wild, D., and Willitsch, S. (2013) Science, 342, 98.

148 Khriachtchev, L., Domanskaya, A., Marushkevich, K., Räsänen, M., Grigorenko, B., Ermilov, A., Andrijchenko, N., and Nemukhin, A. (2009) J. Phys. Chem. A, 113, 8143.

149 Taatjes, C.A., Welz, O., Eskola, A.J., Savee, J.D., Scheer, A.M., Shallcross, D.E., Rotavera, B., Lee, E.P.F., Dyke, 
Stefan Willitsch: Chemistry with controlled ions — Chap. 1 - 2016/5/17 - 10:55 — page 33

J.M., Mok, D.K.W., Osborn, D.L., and Percival, C.J. (2013) Science, 340, 177.

150 Rösch, D., Willitsch, S., Chang, Y.P., and Küpper, J. (2014) J. Chem. Phys., 140, 124202.

151 Bressel, U., Borodin, A., Shen, J., Hansen, M., Ernsting, I., and Schiller, S. (2012) Phys. Rev. Lett., 108, 183003.

152 Mur-Petit, J., Pérez-Ríos, J., Campos-Martínez, J., Hernández, M.I., Willitsch, S., and García-Ripoll, J.J.
(2012) Phys. Rev. A, 85, 022308.

153 Wolf, F., Wan, Y., Heip, J.C., Gebert, F., Shi, C., and Schmidt, P.O. (2016) Nature, 530, 457.

154 Schneider, C., Schowalter, S.J., Chen, K., Sullivan, S.T., and Hudson, E.R. (2014) Phys. Rev. Appl., 2, 034013.

155 Meyer, K.A.E., Pollum, L.L., Petralia, L.S., Tauschinsky, A., Rennick, C.J., Softley, T.P., and Heazlewood, B.R. (2015) J. Phys. Chem. A, 119, 12449. 
Stefan Willitsch: Chemistry with controlled ions - Chap. 1 - 2016/5/17 - 10:55 - page 34

34 